\title{
Extended-range temporal electronic speckle pattern interferometry
}

\author{
Manuel Servin, Abundio Davila, and Juan Antonio Quiroga
}

\begin{abstract}
In recent years the availability of high-speed digital video cameras has motivated the study of electronic speckle pattern interferometry (ESPI) in the time domain. To this end a properly sampled temporal sequence of $N$-fringe patterns is used to analyze the temporal experiment. Samples of temporal speckle images must fulfill the Nyquist criteria over the time axis. When the transient phenomena under study are too fast, the required sampling frequency over time may not be fulfilled. In that case one needs to extend the measuring range of the algorithm used to extract the modulating phase. We analyze how to use short laser pulses or short video acquisition times with fairly long temporal separation among them to estimate the modulating phase of a dynamic ESPI experiment. The only requirement is that the modulating phase being estimated be properly sampled in the spatial domain. (c) 2002 Optical Society of America

OCIS codes: $120.2650,120.6160,120.5050$.
\end{abstract}

\section{Introduction}

Electronic speckle pattern interferometry (ESPI) is a well-established technique that is used for a wide range of mechanical analyses of nonspecular surfaces. Temporal fringe pattern analyses applied to the study of transient phenomena by using electronic speckle pattern interferometry (TESPI) has been an intense research subject in recent years. Even though high-power double-pulse lasers have been used for this purpose, ${ }^{1-3}$ sometimes a quantitative analysis cannot be obtained because of the difficulty of demodulating ESPI fringe patterns containing high-density closed fringes. ${ }^{2}$

In recent years high-speed CCD digital video cameras for the optical laboratory are capable of obtaining more than 45,000 digital pictures per second. ${ }^{2}$ Using such a technology, one may analyze moderately high-speed transient phenomena in a quasicontinuous manner. Among the most notable techniques applied to date have been the temporal

M. Servin (mservin@cio.mx) and A. Davila are with the Centro de Investigaciones en Optica A.C., Apartado Postal 1-948, 37150 Leon Guanajuato, Mexico. J. A. Quiroga is with the Departamento de Optica, Universidad Complutense de Madrid, Ciudad Universitaria S/N, 28040, Madrid, Spain.

Received 3 December 2001; revised manuscript received 1 March 2002.

0003-6935/02/224541-07\$15.00/0

(C) 2002 Optical Society of America unwrapping method devised by Huntley et $a l .^{4,5}$ and the temporal Fourier method applied by Joenathan et al. ${ }^{6-9}$ These temporal techniques are applicable whenever the temporal signal contains less than a half-fringe (less than $\pi$ rad) per sample. If this sampling rate requirement is not fulfilled, the temporal fringes are subsampled and the standard method of temporal analysis ${ }^{4-9}$ is not applicable.

In this paper a method is presented for obtaining useful temporal phase information even in cases in which the required temporal sampling rate of two samples per fringe ${ }^{4-9}$ is not fulfilled. To obtain this reduction in the temporal sampling rate while avoiding phase information losses, the spatial interferogram must be properly sampled in the spatial domain between two successive samples.

\section{Temporal Sampling of Dynamic ESPI Interferograms}

Let us first analyze a typical space-temporal signal given by a standard ESPI interferometer, this threedimensional signal may be modeled according to

$$
\begin{aligned}
I(x, y, t)= & a(x, y)+b(x, y) \cos [\phi(x, y, t) \\
& +\psi(x, y)],
\end{aligned}
$$

where $\phi(x, y, t)$ is the modulating phase, which is related to the physical quantity being studied; $a(x, y)$ is low-frequency background illumination; $b(x, y)$ is the contrast in the speckle, which is also a lowfrequency signal. The speckle phase $\psi(x, y)$ is timeinvariant (assuming no speckle decorrelation during 

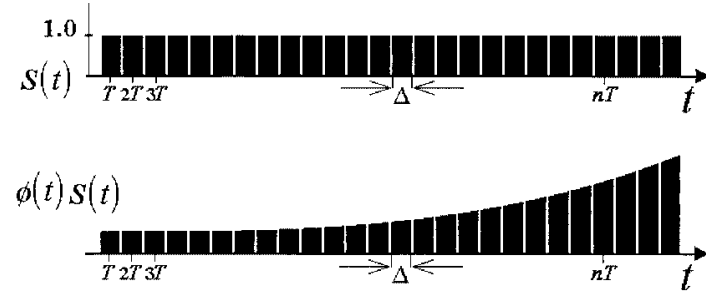

(a)

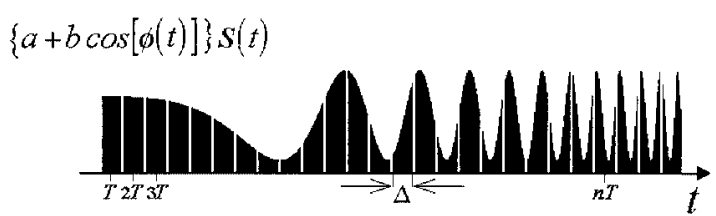

(b)

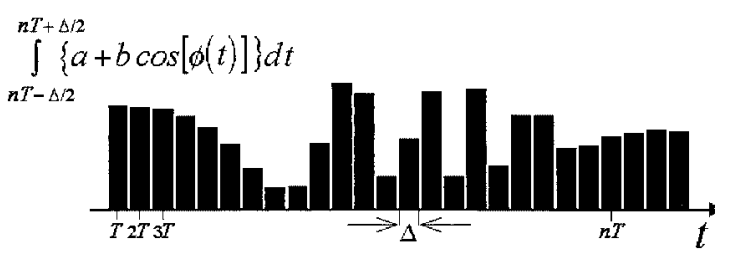

(c)

Fig. 1. Temporal sampling of a signal: (a) thick temporal sampler signal and sampled phase, (b) sampled fringe pattern, (c) integration over the sampling time of the signal.

the experiment) random noise within the range $(-\pi$, $\pi)$. Now our main task is to recover the timevarying phase $\phi(x, y, t)$ information that is proportional to the dynamic quantity being measured.

Figure 1(a) shows a wide-pulse temporal sampling signal $S(t)$. This signal may be represented by

$$
S(t)=\sum_{n=0}^{N-1} \Pi\left(\frac{t-n T}{\Delta}\right)
$$

It is formed by $N$ temporal windows $\Pi(\mathrm{t} / \Delta)$ with a duration $\Delta$ and separated $T$ seconds $(\Delta<T)$

To simplify our analysis let us assume that our phase at any time within the sampling period $T$ may be represented by the first two terms of its Taylor expansion around the sampling time $n T$ [see Figs. 1 (a) and $1(\mathrm{~b})$ ], that is,

$$
\begin{aligned}
I(x, y, t) S(t) \approx & \sum_{n=0}^{N-1}\{a(x, y)+b(x, y) \cos [\phi(x, y, n T) \\
& +\omega(x, y, n T)(t-n T) \\
& +\psi(x, y)]\} \Pi\left(\frac{t-n T}{\Delta}\right) .
\end{aligned}
$$

The signal $\phi(x, y, n T)$ represents the value of the continuous phase $\phi(x, y, t)$ at time $n T$. The signal $\omega(x, y, n T)$ represents the temporal frequency of the signal at time $n T$. Let us assume that the temporal signal is integrated over the time $\Delta$ within the camera's photodetector pixel as depicted in Fig. 1. Then for at a given sampling time $n T$ we collect from the video camera data given by

$$
\begin{aligned}
I(x, y, n T)= & \int_{n T-\Delta / 2}^{n T+\Delta / 2}\{a(x, y) \\
& +b(x, y) \cos [\phi(x, y, n T) \\
& +\omega(x, y, n T)(t-n T)+\psi(x, y)]\} \mathrm{d} t
\end{aligned}
$$

then the following temporal sequence of $N$ pictures is created within the digital memory and may be written as

$$
\begin{aligned}
I_{S}(x, y, t)= & \sum_{n=0}^{N-1} I(x, y, n T) \delta(t-n T) \\
I_{S}(x, y, t)= & \Delta \sum_{n=0}^{N-1}\{a(x, y) \\
& +b(x, y) \operatorname{sinc}\left[\frac{\omega(n T) \Delta}{2}\right] \cos [\phi(x, y, n T) \\
& \left.\left.+\frac{\Delta}{2} \omega(n T)+\psi(x, y)\right]\right\} \delta(t-n T) .
\end{aligned}
$$

As we see from Eq. (5) and Fig. 1(c), if the integrating video time of a pixel equals one period of the temporal signal being sampled, the pixel's contrast falls to zero because of the $\operatorname{sinc}(\cdot)$ function involved. So, if we want ideal temporal samples, the integration time $\Delta$ must be small with reference to the sampling period $T$. Then, for a short integrating time $\Delta$, Eq. (5) may be approximated by

$$
\begin{aligned}
I_{S}(x, y, t)= & \Delta \sum_{n=0}^{N-1}\{a(x, y)+b(x, y) \cos [\phi(x, y, n T) \\
& +\psi(x, y)]\} \delta(t-n T),
\end{aligned}
$$

which is the standard relation for an ideal sampled temporal signal. Note that the temporal twodimensional specklegrams in Eq. (6) do not show any spatial fringes; we see only speckle patterns. However, low-noise fringes do exist along the time coordinate, so the phase demodulation along the time line is usually not very difficult. ${ }^{4-10}$

\section{Temporal-Carrier Phase Estimation}

Looking at Eq. (6), we see that, using only this signal, one cannot demodulate the signal unambiguously along the temporal axis, because from the sequence of images given by Eq. (6) it is not possible to know whether the time-varying speckles correspond to a deformation toward or away from the video camera (in the case of the out-of-plane sensitivity ESPI configuration). There are two ways to deal with this annoyance; one is the introduction of a temporal carrier, and the second involves the use of a second camera whose collected image is optically in quadrature with that used to take the specklegrams in Eq. (6).

For the reader's convenience, let us review the 


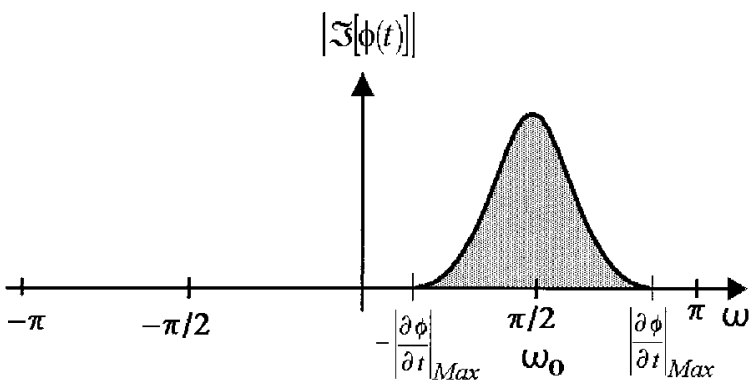

Fig. 2. Spectrum of a typical wideband temporal carrier signal.

phase-estimation technique based on the introduction of a temporal carrier. There are two equivalent ways to introduce a temporal carrier. The one used by Joenathan et al. ${ }^{6-9}$ consists in moving the object being tested toward (or away from) the video camera. The displacement velocity must be greater than the maximum rate of variation of the surface being tested. Another equivalent way to do this is by moving (or phase stepping) the reference surface toward (or away from) the video camera., ${ }^{4,5}$ In both cases the temporal samples will have the following mathematical form:

$$
\begin{aligned}
\frac{I(x, y, n T)}{\Delta}= & a(x, y)+b(x, y) \cos [\phi(x, y, n T) \\
& \left.+\psi(x, y)+\omega_{0} n T\right] .
\end{aligned}
$$

The carrier temporal frequency $\omega_{0}$ must fulfill the following inequality:

$$
\omega_{0}>\left|\frac{\partial \phi(x, y, t)}{\partial t}\right|_{\max },
$$

that is, the temporal carrier must be greater than the maximum temporal rate of change of the phase being measured. The spectrum of a noiseless speckle temporal signal with a temporal carrier of $\pi / 2 \mathrm{rad} /$ period $T$ is depicted schematically in Fig. 2. When this temporal frequency is used, the maximum instantaneous phase change $|\partial \phi / \partial t|_{\max }$ must be less than $\pi / 2$ $\mathrm{rad} /$ period. The main drawback of this approach is that more than half of the theoretically available bandwidth is not used.

In the case of perfectly correlated speckle, the signal given by Eq. (1) along the time coordinate is noiseless except for a random initial phase that is the speckle phase $\psi(x, y)$. Experimentally we will have some amount of speckle decorrelation that translates into a low-energy phase noise in the temporally sampled signal. ${ }^{4-8}$ Temporal fringe decorrelation can be kept under control as shown by Joenathan et al. ${ }^{9}$ whenever unwanted displacements to which the speckle interferometer is not sensitive, such as in plane displacement in out-of-plane sensitive interferometers, or out-of-plane displacement in in-plane sensitive interferometers, gives rise to decorrelation. If this warning is observed, one may have an extremely wide measurable dynamic range of as many as a few centimeters. ${ }^{9}$ Joenathan et al..$^{9}$ were capable of obtaining 600 speckle temporal frames having $\sim 85$ temporal fringes with very little decorrelation. This means that the temporal fringe saving method exposed in this research can be used easily to reduce the temporal sampling demand of fast transient phenomena.

To demodulate temporal-carrier signals, one may proceed as in the case of the spatial-carrier-frequency analysis; one may use small support quadrature filters along the time coordinate (phase-stepping techniques ${ }^{4,5}$ or to digitally store the full time length of the experiment and apply the Fourier techniques in the time coordinate. ${ }^{6-9}$

\section{Temporal-Phase Estimation without a Temporal Carrier}

The main drawback of using a temporal carrier is analogous to the limitation that exists in the case of the spatial carrier, namely, a waste of more than one half of the available frequency spectrum for a given sampling rate. One way to eliminate this limitation is to use two video cameras collecting two in quadrature speckle image ${ }^{10}$ or just one camera in a compact experimental setup similar to that of Somers and van Brug. ${ }^{11}$ In this way one obtains simultaneously from the experimental setup two orthogonal signals given by

$$
\begin{aligned}
I c(x, y, t)= & \Delta \sum_{n=0}^{N-1}\{a(x, y)+b(x, y) \cos [\phi(x, y, n T) \\
& +\psi(x, y)]\} \delta(t-n T), \\
I s(x, y, t)= & \Delta \sum_{n=0}^{N-1}\{a(x, y)+b(x, y) \sin [\phi(x, y, n T) \\
& +\psi(x, y)]\} \delta(t-n T) .
\end{aligned}
$$

Using these $2 N$ signals and an appropriate phaseshifting algorithm, one may estimate that temporal phase changes are at least twice as fast as that obtained with the temporal-carrier approach. One way of detecting the time-varying phase is by using the four-frame algorithm proposed by Brug. ${ }^{10}$ Another way to estimate the phase of this signal is to remove the background components $a(x, y)$ from the temporal set of signals and subtract $a(x, y)$ from Eq. (9). This may be achieved by simply summing up the $2 N$ specklegrams as

$$
a_{e}(x, y)=\frac{1}{2 \Delta N} \sum_{n=0}^{N-1}[I s(x, y, n T)+I c(x, y, n T)]
$$

so that the modulating phase will be given by

$$
\begin{aligned}
\phi_{W}(x, y, n T)+\psi(x, y)= & \arctan \\
& \times\left[\frac{I s(x, y, n T)-a_{e}(x, y)}{I c(x, y, n T)-a_{e}(x, y)}\right], \\
n & =0,1, \ldots, N-1 .
\end{aligned}
$$


In this way one obtains the wrapped modulating phase. Using Eq. (11), one is able to use fully the available bandwidth, which means that one may record as many as $\pi$ rad of temporal-phase change without undersampling the temporal signal. So this approach has a net gain of twice the signal bandwidth for a given frame-grabbing frequency $1 / T$ with reference to the temporal-carrier method.

The last step on this phase analysis is to unwrap the estimated phase [Eq. (11)] along the temporal axis. This may be done by summing up the wrapped-phase differences as ${ }^{4,5}$

$$
\begin{aligned}
\phi e(x, y, N T)= & \sum_{n=0}^{N-2} W\left\{\phi_{W}[x, y,(n+1) T]+\psi(x, y)\right. \\
& \left.-\phi_{W}[x, y, n T]-\psi(x, y)\right\}, \quad(12)
\end{aligned}
$$

where the function $W(\cdot)$ stands for the wrapping operator modulo $2 \pi$. Using Eq. (12), one sums up phase changes with respect to the phase at the previous time slice. As we see from Eq. (12), the phase noise due to the speckle $\psi(x, y)$ is removed because this noise is constant in the time coordinate.

The main drawback of this quadrature configuration comes from use of a more complicated experimental setup because of the need for obtaining two speckle images per temporal sample. As mentioned above, to obtain these two quadrature speckle images, one may use an experimental setup similar to that used by van Brug and Somers. ${ }^{10,11}$

\section{Extended-Range Temporal Interferometry}

In Section 4 a factor of 2 in the temporal dynamic range of the phase was gained by picking up two interferograms in quadrature per each period $T$. In standard temporal-fringe pattern analysis the integration time $\Delta$ in Eq. (3) is a bit less than the sampling period $T$. Therefore, to demodulate temporal-phase signals having a higher bandwidth, one needs a proportionally higher sampling frequency $1 / T$. But, if our system is already limited to $1 / T$ video frames per second, one may still obtain useful phase data whenever the integration time $\Delta$ is proportionally reduced leaving the sampling period $T$ constant. In other words, we must reduce the temporal integration time $\Delta$ at least to half of the fastest temporal fringe. This is done to avoid contrast reduction due to the $\operatorname{sinc}(\cdot)$ function in Eq. (5), which is shown in Fig. 1. Another equally important requirement of the algorithm proposed is that the modulating phase information $\phi(x, y$, $t$ ) be properly sampled in the space domain.

Once the integration time $\Delta$ has been reduced significantly with respect to $T$, the dynamic phase is obtained by using Eqs. (9)-(11). The next step is to find the incremental phase between two successive temporal samples among the $N-1$ incremental phase maps:

$$
\begin{aligned}
\Delta \phi_{W}(x, y, n T)= & W\left\{\phi_{W}[x, y,(n+1) T]\right. \\
& \left.-\phi_{W}(x, y, n T)\right\}, \\
n & =0, \ldots, N-2 .
\end{aligned}
$$

In standard TESPI one would unwrap these phase increments along the time coordinate [Eq. (12)] and obtain the searched phase. However, if the actual phase differences between two samples is greater than $\pi \mathrm{rad}, \Delta \phi_{w}$ will be wrapped and the integration of Eq. (13) is meaningless. Then, to avoid this problem, we propose instead integrating the spatial gradient of these increments denoted by $d_{x}(x, y, n T)$ and $d_{y}(x, y, n T)$. This spatial gradient at time $n T$ is obtained as first-order differences as

$$
\begin{aligned}
d_{x}(x, y, n T)= & W\left[\Delta \phi_{W}(x, y, n T)-\Delta \phi_{W}(x\right. \\
& -1, y, n T)], \\
n= & 0, \ldots, N-2, \\
d_{y}(x, y, n T)= & W\left[\Delta \phi_{W}(x, y, n T)-\Delta \phi_{W}(x, y\right. \\
& -1, n T)], \\
n= & 0, \ldots, N-2 .
\end{aligned}
$$

If the spatial gradient of $\Delta \phi_{w}$ [Eq. (14)] lies within $\pm \pi$, this gradient will be properly sampled along the time coordinate. Then we may sum up these signals along the time coordinate and obtain the accumulated first-order differences $D_{x}(x, y, n T)$ and $D_{y}(x, y$, $n T)$ as

$$
\begin{gathered}
\phi(x, y, N T)-\phi(x-1, y, N T)=D_{x}(x, y, N T) \\
=\sum_{n=0}^{N-2} d_{x}[x, y, n T], \\
\phi(x, y, N T)-\phi(x, y-1, N T)=D_{y}(x, y, N T) \\
=\sum_{n=0}^{N-2} d_{y}[x, y, n T] .
\end{gathered}
$$

Finally we obtain the searched phase by finding the estimated phase $\phi e(x, y, N T)$ as the minimizer of the following cost function ${ }^{10}$ :

$$
\begin{aligned}
U(\phi e)= & \sum_{(x, y) \in P}\{[\phi e(x, y, N T)-\phi e(x-1, y, N T) \\
& \left.-D_{x}(x, y, N T)\right]^{2}+[\phi e(x, y, N T) \\
& \left.\left.-\phi e(x, y-1, N T)-D_{y}(x, y, N T)\right]^{2}\right\},
\end{aligned}
$$

where $P$ is the two-dimensional lattice of valid phase data. The function $\phi e(\cdot)$ is therefore the accumulated phase change during the entire experiment. Computationally the simplest way to obtain the estimated phase $\phi e(x, y, N T)$ that minimizes Eq. (16) is by use of a fixed-step gradient descent:

$$
\begin{aligned}
\phi e(x, y, N T)^{k+1}= & \phi e(x, y, N T)^{k} \\
& -\left.\frac{\partial U}{\partial \phi e(x, y, N T)}\right|_{k}, \\
k & =0,1,2, \ldots .
\end{aligned}
$$

Although this is a simple algorithm its computational efficiency is poor. A collection of algorithms minimizing this cost function is given in Ref. 12 . 


\section{Discussion}

As we see from Eq. (14) the proposed extended temporal fringe analysis is limited to true spatial phase gradients within the range of $(0,2 \pi)$ rad per pixel. These spatial limitations may be stated as

$$
\left|\begin{array}{l}
\Delta \phi(x, y, n T)-\Delta \phi(x-1, y, n T) \\
\Delta \phi(x, y, n T)-\Delta \phi(x, y-1, n T)
\end{array}\right|<2 \pi,
$$

Although in the standard temporal analyses of fringe patterns we were limited to having less than a half temporal fringe between samples, using the method proposed here, we may significantly increase that range. However, when several temporal fringes exist within a period $T$ the integration time $\Delta$ must be short enough to include only less than a half temporal fringe. If a short temporal integration time $\Delta$ with respect to the sampling time $T$ does not collect enough light, high-energy pulsed lasers may be needed.

As can be seen from this discussion, a desired gain in the temporal measuring range must be followed by a proportional reduction in the temporal integration time $\Delta[$ Eq. (3)] as well as a proportional increase in the spatial resolution of the CCD used to obtain the interferograms. In this way we are interchanging a less demanding temporal sampling rate by a proportionally higher requirement in spatial resolution. One analogy to the extended TESPI technique proposed here is temporal speckle shearing interferometry. ${ }^{10,11,13}$ In temporal speckle shearing interferometry one unwraps along the time coordinate the value of the phase gradient, and one obtains the final modulating phase change throughout the experiment by spatial integration.

As shown in Section 7, using this technique, we still may handle some amount of phase discontinuity among neighborhood pixels that is limited to the range of $(-\pi, \pi)$ between two successive samples. In the worst case of having a sharp phase discontinuity the temporal limitation of the method proposed here is the same as for standard temporal interferometry. ${ }^{4-9}$

\section{Numerical Experiment}

We have simulated on a PC the extended ESPI technique described above. We have modeled the timevarying especklegrams as

$$
\begin{aligned}
I c(x, y, t)= & 128+20 \cos \left[100 t \exp \left(-r^{2} / \sigma^{2}\right)+0.3 t x\right. \\
& +\psi(x, y)]+10 r n d(x, y, t), \\
I s(x, y, t)= & 128+20 \sin \left[100 t \exp \left(-r^{2} / \sigma^{2}\right)+0.3 t x\right. \\
& +\psi(x, y)]+10 r n d(x, y, t),
\end{aligned}
$$

where the speckle phase $\psi(x, y)$ is a computergenerated random variable within the range of $(-\pi$, $\pi) \mathrm{rad}$. The function $\operatorname{rnd}(x, y, t)$ is a uniformly distributed random variable within the $(0,1)$ range. This $r n d(\cdot)$ function simulates a temporal speckle decorrelation noise and the electronic noise. The small time-varying spatial carrier $0.3 t x$ was introduced to point out only that phase tilt is also pre-

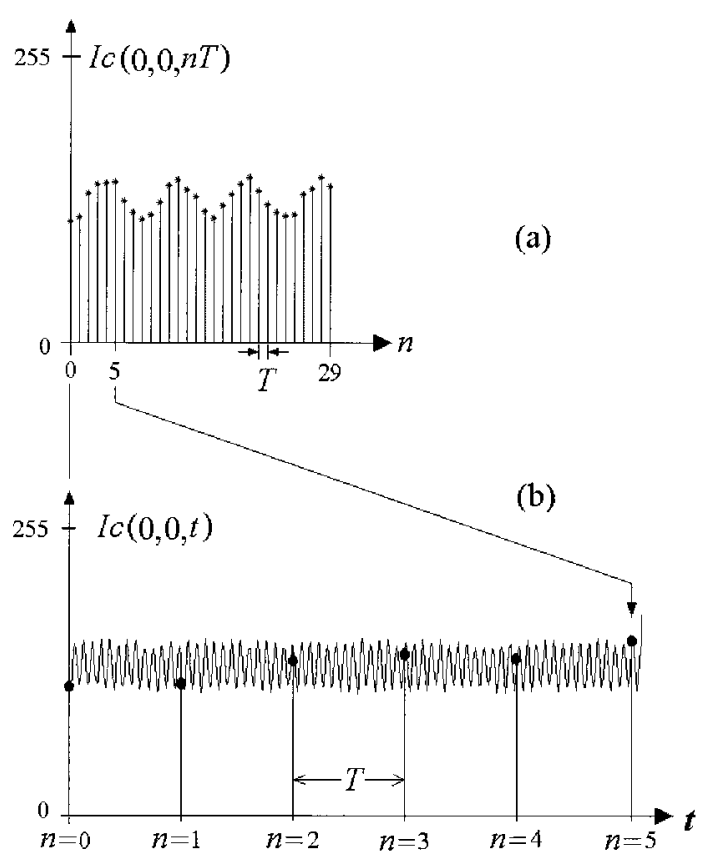

Fig. 3. (a) Undersampled signal, (b) temporal zoom of the first five samples showing also the continuous original signal for comparison purposes.

served in the estimated phase. The signals in Eq. (19) are sampled in the spatial domain with a regular array of $128 \times 128$ pixels by using 256 gray levels. This temporal signal is then sampled in the time domain as

$$
\begin{aligned}
& I c(x, y, t)=\sum_{n=0}^{29} I c(x, y, n) \delta(t-n), \\
& I s(x, y, t)=\sum_{n=0}^{29} I s(x, y, n) \delta(t-n) .
\end{aligned}
$$

Figure 3(a) shows the 30 temporal samples used in this numerical experiment as given at pixel $I c(0,0$, $n T$ ) at the center of the Gaussian in Eqs. (19) and (20). At that point the sampled signal is heavily undersampled as shown in Fig. 3(b) where we have represented the temporal signal $\operatorname{Ic}(0,0, t)[\mathrm{Eq} .(19)]$ in greater detail along with its first six samples.

Figure 4(a) shows the mesh graph corresponding to the accumulated phase differences $D_{x}(x, y, n T)$ and $D_{y}(x, y, n T)$ according to Eq. (15). The spatially integrated phase, which is obtained as the minimizer of Eq. (16), is shown in Fig. 4(b). We can see the high dynamic range $(\sim 1000 \pi \mathrm{rad})$ obtained by using only 30 speckle images [Eq. (20)]. Using a standard temporal ESPI, one would need at least 1000 images to obtain the same measuring range, and, using a carrier temporal ESPI, one would need more than 2000 specklegrams. So in this particular case we have reduced the sampling frequency by a factor greater than 66 times with reference to the carrier temporal analysis.

Another way to see this sampling-frequency reduction is by looking at the wrapped phase between two 


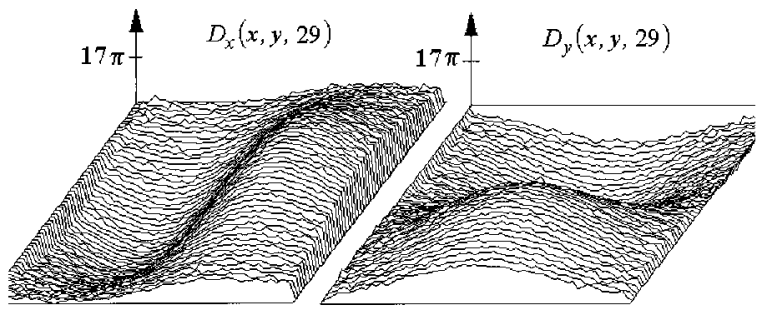

(a)

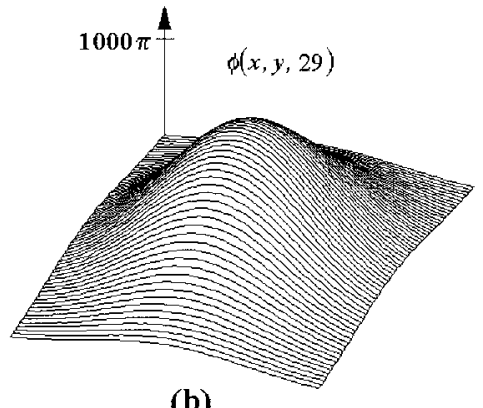

(b)

Fig. 4. (a) Accumulated first-order differences along the $x$ and $y$ directions, (b) spatially integrated signal of the accumulated phase gradient.

successive temporal samples. In standard TESPI one cannot exceed half of a fringe per temporal sampling period ( $\pm \pi \mathrm{rad})$ as seen in Fig. 5(a). On the other hand, using the extended TESPI proposed, one may easily have several fringes [as seen in Fig. 5(b)] per sampling period. Extending the spatial resolution of the speckle images, one may increase the measuring range of the extended TESPI technique by a proportional amount. That is, for this example, [Eqs. (19)-(20)] increasing the spatial resolution from $128 \times 128$ pixels to $256 \times 256$ pixels, one may extend twice the number of fringes per temporal sample. We conclude that the skipped temporal fringes (Fig. 3) are stored as spatial fringes (Fig. 5) so in the end no information is lost.

In Fig. 6 we show that the proposed extended TESPI technique may still be used when phase discontinuity is present. In this case the amount of phase discontinuity allowed has the same limitation as in the case of standard temporal-phase analysis,

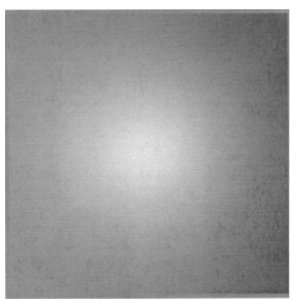

(a)

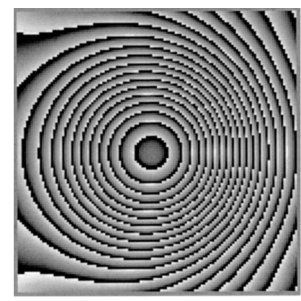

(b)
Fig. 5. (a) Maximum phase variation between two successive temporal samples by using standard TESPI, (b) phase variation between successive samples by using the proposed extended TESPI technique.

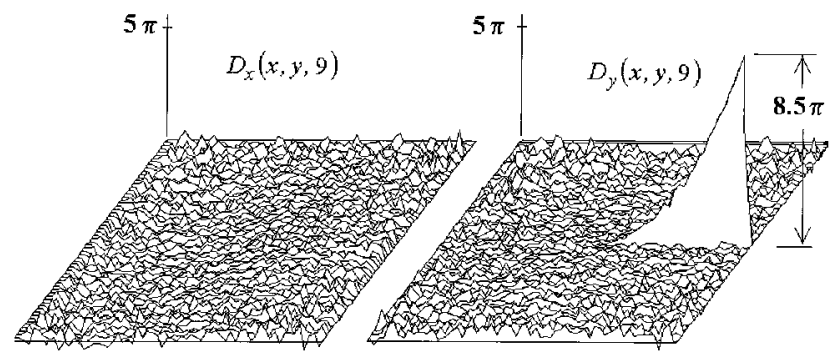

(a)

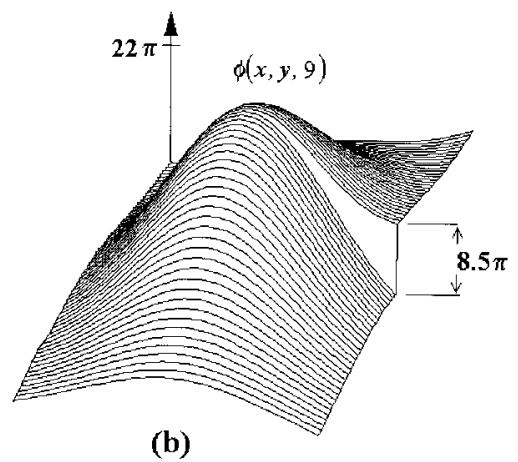

Fig. 6. Temporal analysis of a varying phase with a phase jump: (a) accumulated first-order differences along the $x$ and $y$ directions, (b) spatially integrated signal of the accumulated phase gradient.

which is limited to a maximum of $\pm \pi$ rad between two successive samples. In Fig. 6 we used 10 temporal samples so that the maximum recordable phase jump between sample zero and sample nine is a bit less than $9 \pi$ rad. But the smooth part of the phase may easily exceed such a limit as shown in Figs. 4 and 5.

\section{Conclusion}

A new extended range temporal ESPI technique has been presented. The extended TESPI method presented has a wider range of measurement than the standard temporal ESPI when a smooth modulation phase is recorded. Standard TESPI where two orthogonal specklegrams per temporal image are used is limited to recording as much as a half temporal and/or a half spatial fringe per time frame. In this extended TESPI method one may have several spatial fringes per frame. The limitation on the extended ESPI method resides in the maximum number of spatial fringes that can appear between two temporal samples; this number is limited by the resolution of the CCD video camera.

This extended ESPI technique is applicable when two orthogonal speckle images are used taken in parallel by using two cameras in the experimental setup (or just one camera as in Ref. 11). As mentioned above, when this system is used, the temporal Nyquist limit among the recorded frames is no longer restricted to less than a half temporal fringe. However, the spatial phase gradient per temporal 
step must not exceed $\pm \pi \mathrm{rad} /$ pixel for each temporal sample.

In other words, to extend the measuring range of TESPI we propose calculating the temporal variations of the spatial phase gradient. The temporal variation of the spatial phase gradient is then summed up along the time coordinate and integrated by least squares at the end of the entire experiment. The integration of the accumulated spatial phase gradient will give us the total amount of phase change during the experiment.

The two main drawbacks of the extended TESPI technique proposed here is that it is mostly applied to the smooth space modulating phase and the need to take two simultaneous speckle images in quadrature.

We acknowledge the valuable support of the Mexican Council of Science and Technology (CONACYT) as well as the support of the European Union Project INDUCE.BRPR-CT97-0805.

\section{References}

1. A. Fernandez, A. J. Moore, C. Perez-Lopez, A. F. Doval, and J. Blanco-Garcia, "Study of transient deformations with pulsed TV holography: application to crack detection," Appl. Opt. 36, 2059-2065 (1997).

2. A. J. Moore, D. P. Hand, J. S. Barton, and J. D. C. Jones, "Transient deformation measurement with electronic speckle pattern interferometry and a high-speed camera," Appl. Opt. 38, 1159-1162 (1999).

3. A. Fernandez, J. Blanco-Garcia, A. F. Doval, J. Bugarin, B. V. Dorrio, C. Lopez, J. M. Alen, M. Perez-Amor, and J. L. Fernandez, "Transient deformation measurement by doublepulsed-subtraction TV holography and the Fourier transform method," Appl. Opt. 37, 3441-3446 (1998).
4. J. M. Huntley, G. H. Kaufmann, and D. Kerr, "Phase-shifted dynamic speckle pattern interferometry at $1 \mathrm{kHz}$," Appl. Opt. 38, 6556-6563 (1999).

5. J. M. Huntley, "Automated analysis of speckle interferograms," in Digital Speckle Pattern Interferometry and Related Techniques, P. K. Rastogi, ed. (Wiley, New York, 2001).

6. C. Joenathan, B. Franze, P. Haible, and H. J. Tiziani, "Novel temporal Fourier transform speckle pattern shearing interferometer," Opt. Eng. 37, 1791-1795 (1998).

7. C. Joenathan, B. Franze, P. Haible, and H. Tiziani, "Large in-plane displacement measurement in dual beam speckle interferometry using temporal phase measurement," J. Mod. Opt. 45, 1975-1984 (1998).

8. C. Joenathan, B. Franze, P. Haible, and H. J. Tiziani, "Speckle interferometry with temporal phase evaluation for measuring large-object deformation," Appl. Opt. 37, 2609-2614 (1998).

9. C. Joenathan, P. Haible, and J. Tiziani, "Speckle interferometry with temporal phase evaluation: influence of decorrelation, speckle size, and nonlinearity of the camera," Appl. Opt. 38, 1169-1178 (1999).

10. H. van Brug, "Temporal phase unwrapping and its application in shearography systems," Appl. Opt. 37, 6701-6706 (1998).

11. P. A. A. M. Somers and H. van Brug, "A single camera, dual image real-time-phase-stepped shearing speckle interferometer," in Fringe'01, Fourth International Workshop on Automatic Processing of Fringe Patterns, W. Osten and W. Juptner, eds. (Elsevier, Paris, 2001), pp. 573-580.

12. D. C. Ghiglia and L. A. Romero, "Robust two-dimensional weighted and unweighted phase unwrapping that uses fast transforms and iterative methods," J. Opt. Soc. Am. 11, 107117 (1994).

13. R. A. Martinez-Celorio, A. Davila, G. H. Kaufmann, and G. Mendiola, "Extension of the displacement measurement range for electronic speckle-shearing pattern interferometry using carrier fringes and a temporal phase unwrapping method," Opt. Eng. 39, 751-757 (2000). 